\title{
A COMPARISON OF THREE EQUALIZATION TECHNIQUES FOR INET-FORMATTED SOQPSK-TG
}

\author{
Michael Rice \\ Brigham Young University \\ Md. Shah Afran \\ Mohammad Saquib \\ University of Texas at Dallas \\ Arlene Cole-Rhodes \\ Farzad Moazzami \\ Morgan State University
}

\begin{abstract}
This paper demonstrates the effectiveness of the zero-forcing, minimum mean-squared error, and constant-modulus equalizers in improving the BER performance of iNET-formatted SOQPSK-TG. The equalization algorithms leverage the existence of known bit sequences in the preamble and ASM fields of the iNET packet to realize data-aided equalizers. The effectiveness of these equalization techniques over ten test channels, derived from channel sounding experiments at Edwards AFB, was evaluated. The BER curves for nine out of the ten test channels display the desirable "waterfall" shape. The BER curve for the remaining test channel displays a "BER floor." Fortunately, the "BER floor" is low enough to allow error correcting codes, such as the iNET LDPC code, to correct the errors and provide virtually error-free performance.
\end{abstract}

\section{INTRODUCTION}

Multipath interference occurs when the radio signal form the airborne transmitted arrives at a ground-based receiver via multiple propagation paths. Usually, one of the propagation paths is the line-of-sight propagation path whereas the others are due to reflections. Multipath interference continues to be the dominant cause of link outages in aeronautical telemetry.

Approved for public release; distribution is unlimited. 412TW-PA-14264 


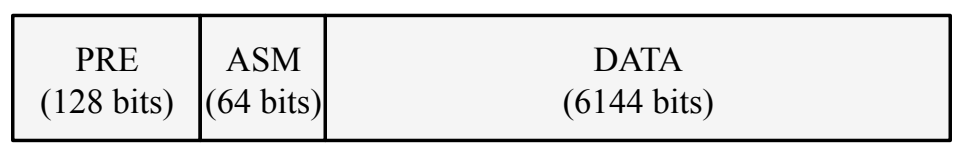

Figure 1: The iNET packet structure used in this paper.

Equalization, using the constant modulus algorithm (CMA), in the context of aeronautical telemetry has been investigated before $[1,2,3,4]$. However, laboratory tests with hardware prototypes produced less than compelling results [4]; these results have raised questions on the applicability to aeronautical telemetry of purely blind equalization algorithms.

Here we investigate a data-aided approach to equalization. In data-aided equalization, the equalizer filter coefficients may be computed from the multipath channel coefficients. The question is, "how does the receiver know what the channel impulse response is?"

The answer to this question lies in the iNET packet structure. iNET-formatted transmissions include a 128-bit preamble and 64-bit attached sync marker (ASM) preceding a block of data bits (at least 6144 bits: an LDPC codeword): see Figure 1. Because the preamble and ASM bits are known, the receiver can compare the received signal to a locally stored copy of the SOQPSK-TG signal corresponding to the preamble and ASM bit fields. This comparison is capable of producing estimates of the frequency offset, noise variance, and multipath channel coefficients [5]. And from the multipath channel coefficient estimates, the equalizer filter coefficients may be obtained.

In this paper we evaluate the performance of three popular equalization algorithms: the zeroforcing (ZF) equalizer, the minimum mean-squared error (MMSE) equalizer, and the CMA equalizer. The ZF and MMSE equalizers are data-aided equalizers. Traditionally, the CMA equalizer is a completely blind equalizer; but here we use the MMSE equalizer filter coefficients to initialize the CMA equalizer. Relative to the traditional "center-tap initialization," the MMSE-initialized CMA equalizer as a shorter convergence time and lower bit error rate.

\section{SYSTEM-LEVEL DESCRIPTION}

The bit sequence for iNET is organized as outlined in Figure 1. The preamble sequence (PRE) is $\mathrm{CD} 98_{\text {hex }}$ repeated eight times [6, p. 48]. The preamble field is followed by the attached sync marker (ASM) field defined as $034776 \mathrm{C} 7272895 \mathrm{~B} 0_{\text {hex }}$. The DATA field is 6144 randomized data bits. (These bits correspond to a single LDPC codeword in the coded system. Here, we evaluate the uncoded bit error rate after equalization.)

The transmitted signal is SOQPSK-TG whose input bit stream is summarized in Figure 1. The signal propagates through a frequency selective channel and experiences a frequency offset as well as the addition of additive white Gaussian noise.

The received signal is filtered, down-converted to I/Q baseband, and sampled (not necessarily in 


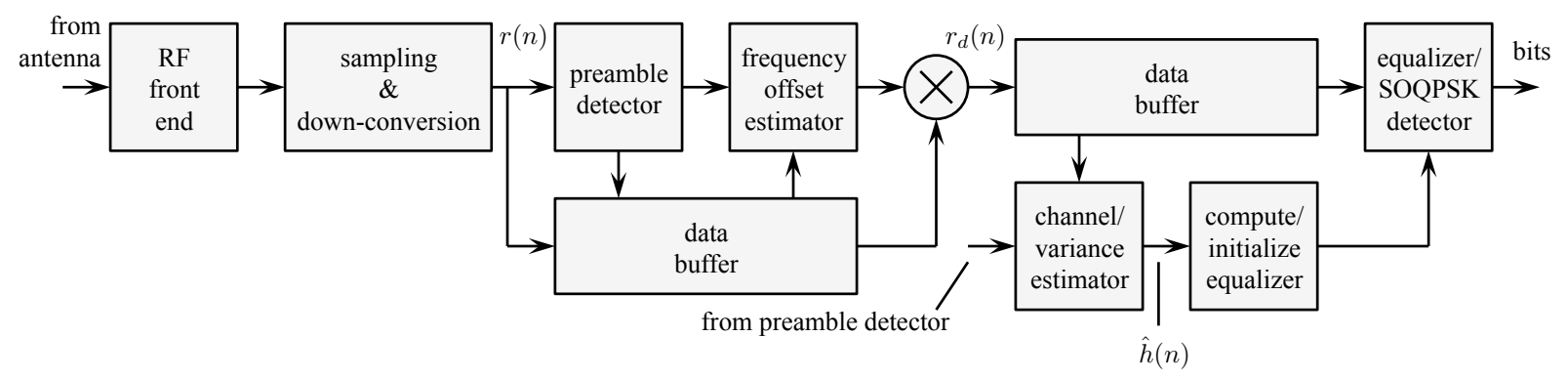

Figure 2: The data packet format and high-level signal processing explored in this paper.

that order) using standard techniques. The resulting sequence of received samples is

$$
r(n)=\left[\sum_{k=-N_{1}}^{N_{2}} h(k) s(n-k)\right] e^{j \omega_{0} n}+w(n),
$$

where $h(n)$ is the impulse response of the equivalent discrete-time channel with support on $-N_{1} \leq$ $n \leq N_{2}, \omega_{0}$ rads/sample is the frequency offset, and $w(n)$ is a complex-valued zero-mean Gaussian random process with variance $\sigma_{w}^{2}$. The focus of this paper is on the application of equalization techniques to the I/Q baseband samples. Because SOQPSK-TG is a nonlinear modulation, the equalizer cannot operate on the symbols in the same way it does for linear modulation (cf., [7, Chapter 9]). Consequently, the equalizer must operate on the samples of SOQPSK-TG, similar to the way fractionally spaced equalizers operate.

Because the preamble and ASM bits are known, the samples corresponding to the preamble and ASM bits are used to estimate the frequency offset, channel impulse response, and, for the MMSE equalizer, the noise variance. Before these tasks can be accomplished, the start of the samples corresponding to the preamble bits in the received signal must be known. This is accomplished by the preamble detector block, whose algorithm is based on the detection algorithm described in [8]. Once the start of the preamble is known, the frequency offset is estimated using the algorithms described in [5]. The frequency offset is used with a complex-exponential to derorate the received data to remove the frequency offset. The derorated data $r_{d}(n)$ are used to estimate the channel and noise variance as described in [5]. The channel estimates $\hat{h}(n)$, for $-N_{1} \leq n \leq N_{2}$, are used to compute the ZF and MMSE equalizer filter coefficients. For the CMA equalizer, the channel estimates $\hat{h}(n)$ are used to initialize the adaptive filter whose update is based on the CMA.

\section{THE EQUALIZATION ALGORITHMS}

The equalizers operate in the system configuration shown in Figure 3. [cf., Figure 2]. Here, the derotated samples $r_{d}(n)$ are equalized using a length $L_{1}+L_{2}+1$ FIR filter defined by the impulse response $c(n)$ for $-L_{1} \leq n \leq L_{2}$ to produce the output

$$
y(n)=\sum_{m=-L_{1}}^{L_{2}} c(m) r_{d}(n-m) .
$$



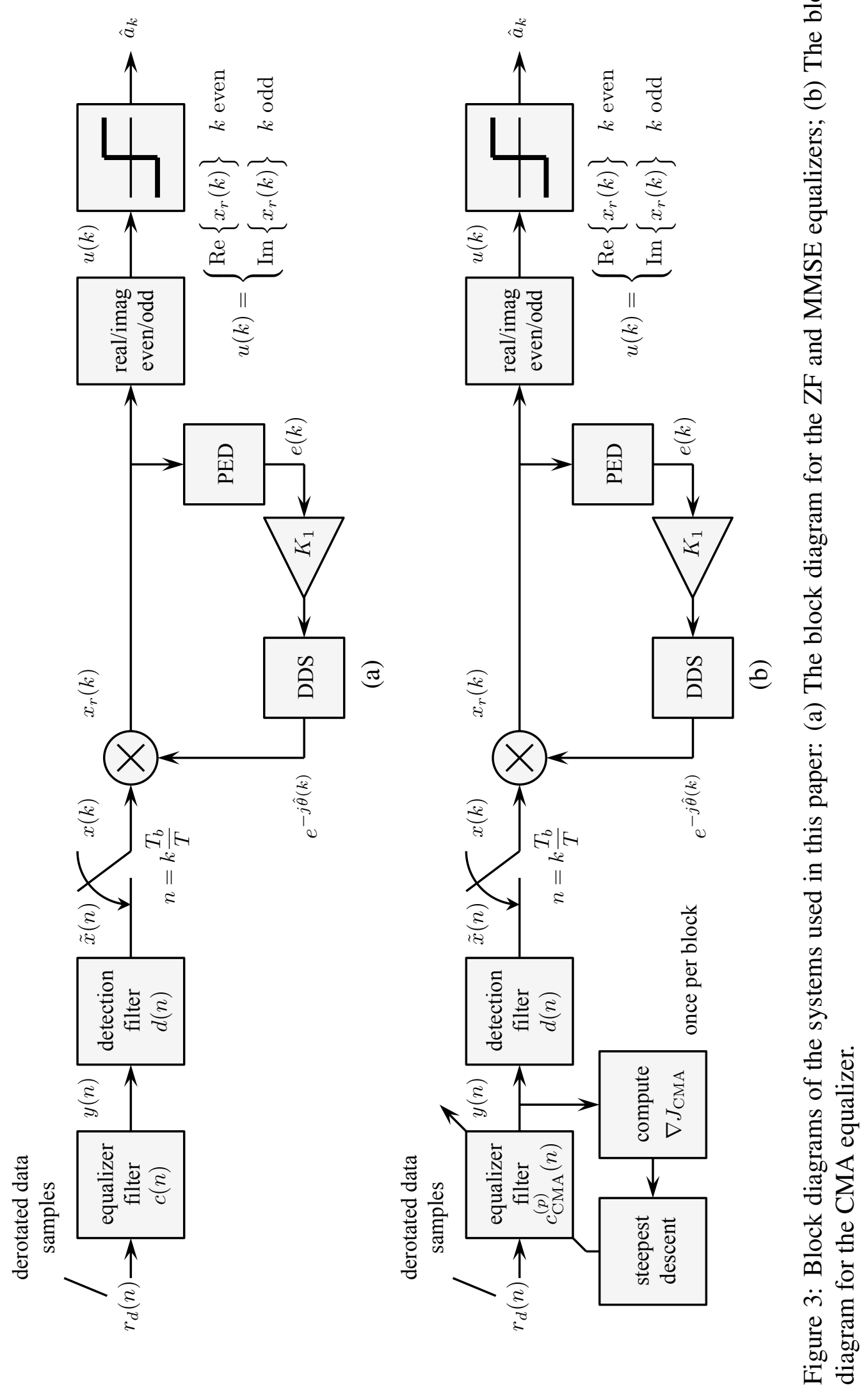
The equalizer output forms the input to the well-known symbol-by-symbol SOQPSK detector comprising a detection filter operating at $N=T_{b} / T$ samples/bit and a decision process, operating on the decision variable $u(k)$ at 1 sample/bit. This detector, based on an offset QPSK approximation of SOQPSK-TG, is described in more detail in [9, 10]. The detectors of Figure 3 also include a phase lock loop (PLL). The PLL is required to track out any residual phase increments due to frequency offset estimation errors. A timing loop is not required because timing offsets are part of the channel estimate $\hat{h}(n)$. In what follows, we will organize the equalizer filter coefficients into $\left(L_{1}+L_{2}+1\right) \times 1$ vectors as follows:

$$
\mathbf{c}_{\mathrm{ZF}}=\left[\begin{array}{c}
c_{\mathrm{ZF}}\left(-L_{1}\right) \\
\vdots \\
c_{\mathrm{ZF}}(0) \\
\vdots \\
c_{\mathrm{ZF}}\left(L_{2}\right)
\end{array}\right], \quad \mathbf{c}_{\mathrm{MMSE}}=\left[\begin{array}{c}
c_{\mathrm{MMSE}}\left(-L_{1}\right) \\
\vdots \\
c_{\mathrm{MMSE}}(0) \\
\vdots \\
c_{\mathrm{MMSE}}\left(L_{2}\right)
\end{array}\right], \quad \mathbf{c}_{\mathrm{MMSE}}^{(p)}=\left[\begin{array}{c}
c_{\mathrm{CMA}}^{(p)}\left(-L_{1}\right) \\
\vdots \\
c_{\mathrm{CMA}}^{(p)}(0) \\
\vdots \\
c_{\mathrm{CMA}}^{(p)}\left(L_{2}\right)
\end{array}\right] .
$$

The superscript " $(p)$ " for the CMA equalizer coefficients is the update index and is explained below.

The ZF equalizer is a filter that is the best length- $\left(L_{1}+L_{2}+1\right)$ FIR approximation to the "inverse" of the channel. That is, $c(n)$ is chosen so that

$$
\hat{h}(n) * c(n) \approx \delta\left(n-n_{0}\right)
$$

for $-L_{1} \leq n \leq L_{2}$ where "best" minimizes the least squares error. The vector of filter coefficients defined by this criterion is given by [11]

$$
\mathbf{c}_{\mathrm{ZF}}=\left(\mathbf{H}^{\dagger} \mathbf{H}\right)^{-1} \mathbf{H}^{\dagger} \mathbf{u}_{n_{0}}
$$

where

$$
\mathbf{u}_{n_{0}}=\left[\begin{array}{c}
0 \\
\vdots \\
0 \\
1 \\
0 \\
\vdots \\
0
\end{array}\right] n_{0}-1 \text { zeros }
$$

and $\mathbf{H}$ is the $\left(N_{1}+N_{2}+L_{1}+L_{2}+1\right) \times\left(L_{1}+L_{2}+1\right)$ convolution matrix formed by the estimates of the channel impulse response:

$$
\mathbf{H}=\left[\begin{array}{cccc}
\hat{h}\left(-N_{1}\right) & & & \\
\hat{h}\left(-N_{1}+1\right) & \hat{h}\left(-N_{1}\right) & & \\
\vdots & \vdots & \ddots & \\
\hat{h}\left(N_{2}\right) & \hat{h}\left(N_{2}-1\right) & & \hat{h}\left(-N_{1}\right) \\
& \hat{h}\left(N_{2}\right) & \hat{h}\left(-N_{1}+1\right) \\
& & \vdots \\
& & & \hat{h}\left(N_{2}\right)
\end{array}\right] .
$$


The minimum mean-squared error (MMSE) equalizer is a filter that minimizes the mean squared error

$$
\mathcal{E}=\mathrm{E}\left\{|s(n)-r(n) * c(n)|^{2}\right\}
$$

The solution is given by a form of the Wiener-Hopf equations [11]. The $\left(L_{1}+L_{2}+1\right) \times 1$ vector of MMSE equalizer filter coefficients are

$$
\mathbf{c}_{\mathrm{MMSE}}=\left[\mathbf{G G}^{\dagger}+\frac{\hat{\sigma}_{w}^{2}}{\sigma_{s}^{2}} \mathbf{I}_{L_{1}+L_{2}+1}\right]^{-1} \mathbf{g}^{\dagger},
$$

$\mathbf{G}$ is the $\left(L_{1}+L_{2}+1\right) \times\left(N_{1}+N_{2}+L_{1}+L_{2}+1\right)$ matrix

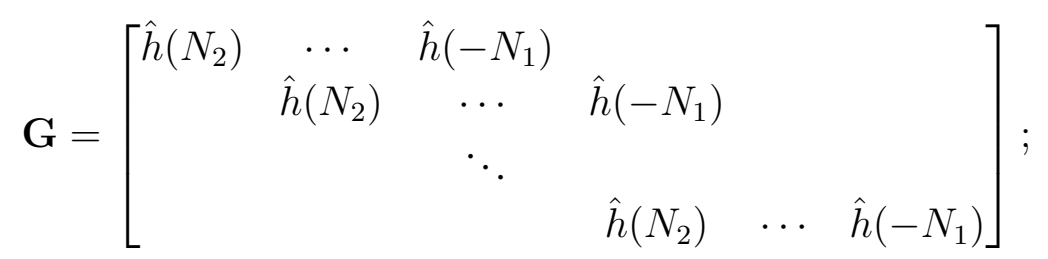

$\mathbf{g}$ is the $1 \times\left(L_{1}+L_{2}+1\right)$ vector given by

$$
\mathbf{g}=\left[\begin{array}{lll}
\hat{h}\left(L_{1}\right) & \cdots & \hat{h}\left(-L_{2}\right)
\end{array}\right]
$$

where it is understood that $h(n)=0$ for $n<-N_{1}$ or $n>N_{2}$ (how many zeros need to be prepended and appended depends on the relationship between $L_{1}$ and $N_{2}$ and the relationship between $L_{2}$ and $N_{1}$ ); and $\sigma_{s}^{2}$ is the signal power. This solution is based on the assumption that SOQPSK-TG samples corresponding to a sample rate of 2 samples/bit are approximately uncorrelated. The impact of this approximation is discussed in [12].

The CMA equalizer is outlined in Figure 3 (b). As with the ZF and MMSE equalizers of Figure 3 (a), the length- $\left(L_{1}+L_{2}+1\right)$ FIR equalizer filter precedes the SOQPSK-TG symbol-by-symbol detector. Unlike the ZF and MMSE equalizers of Figure 3 (a), the CMA equalizer is an adaptive filter whose coefficients minimize the cost function $J_{\mathrm{CMA}}$ given by

$$
J_{\mathrm{CMA}}(y(n))=\mathrm{E}\left\{\left(|y(n)|^{2}-R_{2}\right)^{2}\right\}
$$

where

$$
R_{2}=\frac{\mathrm{E}\left\{|s(n)|^{4}\right\}}{\mathrm{E}\left\{|s(n)|^{2}\right\}} .
$$

The CMA cost function measures the departure of the equalizer output from a circle of radius $R_{2}$, but does so without any phase information.

Using the steepest descent algorithm to drive the adaptation, which occurs once per packet here, the $\left(L_{1}+L_{2}+1\right) \times 1$ vector of filter coefficients for packet $p+1$ is an updated version of the filter coefficients used for packet $p$ :

$$
\mathbf{c}_{\mathrm{CMA}}^{(p+1)}=\mathbf{c}_{\mathrm{CMA}}^{(p)}-\mu \nabla J_{\mathrm{CMA}}
$$




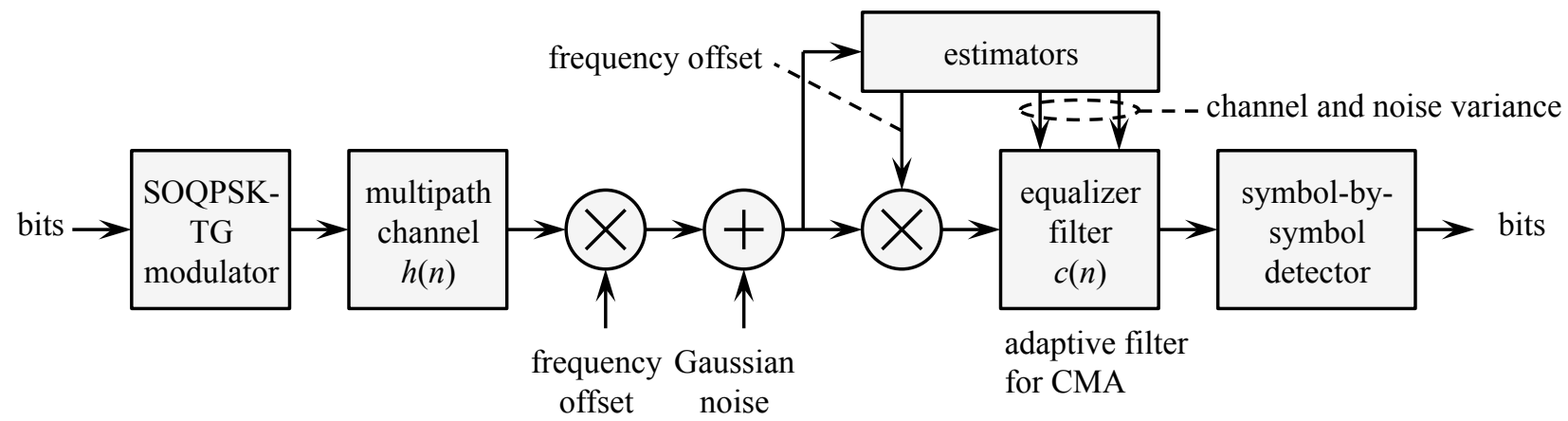

Figure 4: A block diagram of the simulation procedure.

where $\mu>0$ is the step size and where

$$
\nabla J_{\mathrm{CMA}}=\mathrm{E}\left\{2\left[y(n) y^{*}(n)-R_{2}\right] y(n) \mathbf{r}^{*}(n)\right\}
$$

with

$$
\mathbf{r}^{*}(n)=\left[\begin{array}{c}
r_{d}^{*}\left(n+L_{1}\right) \\
\vdots \\
r_{d}^{*}(n) \\
\vdots \\
r_{d}^{*}\left(n-L_{2}\right)
\end{array}\right] .
$$

A critical issue for any adaptive filter is convergence: both how long and to what state. Here, we initialize the CMA equalizer filter with the MMSE filter coefficients. That is

$$
\mathbf{c}_{\mathrm{CMA}}^{(0)}=\mathbf{c}_{\mathrm{MMSE}}
$$

given by (7). Not only does this reduce convergence time, but also it helps the adaptive filter converge to a state the produces a lower bit error rate than that achievable with center-tap-initialization.

\section{PERFORMANCE RESULTS}

The bit error rate (BER) performance of the three equalization techniques was assessed using the simulation environment outlined in Figure 4. The simulation parameters were the following:

1. The payload data rate was equivalent to $10 \mathrm{Mbits} / \mathrm{s}$ (the equivalent "over-the-air" bit rate was $10.3125 \mathrm{Mbits} / \mathrm{s}$ ). The iNET-formatted SOQPSK-TG signal and channel were generated at an equivalent sample rate of 2 samples/bit.

2. Because the channel estimator does not know the true length of the channel, the estimator used values for $N_{1}$ and $N_{2}$ larger than any of the test channels. These values were $N_{1}=12$ and $N_{2}=25$ samples. 
3. The equalizers used $L_{1}=4 \times N_{1}=48$ samples and $L_{2}=4 \times N_{2}=100$ samples. Thus the length of equalizer filter was 149 samples.

4. The CMA equalizer filter is an adaptive filter. After initializing the filter with the MMSE equalizer filter coefficients, the CMA filter was allowed to converge for 100 packets before errors were counted.

5. The simulations were performed over 10 representative channels derived from channel sounding measurements conducted at Edwards AFB under the M4A program [13]. The test channels are summarized in Table 1 and the corresponding frequency-domain plots are shown in Figure 5.

The simulated BER performance is shown in Figures 6 - 10. In all cases we observe that the ZF equalizer has the worst performance and that the MMSE and CMA equalizer have about the same performance. (The lone exception is the simulation results for test channel 9 shown in Figure 10 where for high $E_{b} / N_{0}$, all three equalizer achieve the same performance. This is to be expected because test channel 9 is rather benign [see Figure 5].) The fact that the ZF equalizer has the worst performance is not surprising. The ZF equalizer simply "inverts the channel" (this phrase derives from the frequency domain point of view). For channels with nulls, the "inversion" restores the frequency content of the desired signal in the frequency band surrounding the null. This restoration also amplifies the noise power in the same frequency band. The end result is a phenomenon known as "noise amplification:" the distortion due to the multipath channel is corrected, but the signal-to-noise ratio (greatly) decreased. In contrast the MMSE and CMA equalizers take a more measured approach to "channel inversion" and balance the impact of residual multipath distortion and amplified noise on their respective cost functions [mean squared error (6) for the MMSE equalizer and $J_{\mathrm{CMA}}(8)$ for the CMA equalizer].

Another interesting feature of the simulation results is that all test channels, except test channel 2, display the "waterfall" shape; a large decrease in BER as $E_{b} / N_{0}$ increases above a certain value. Systems characterized by BER vs. $E_{b} / N_{0}$ curves with the "waterfall" shape are those for which equalization and additional link margin can achieve a desired reliability level. The good news here is that for nine out of the ten cases, reliable communications is possible. Test channel 2 is a counter example. Here, the BER performance seems to "flatten out" as $E_{b} / N_{0}$ increases. That is, the BER curve is characterized by a "BER floor" rather than the "waterfall" shape. A "BER floor" is undesirable because it means that no matter how much $E_{b} / N_{0}$ is increased, the system designer cannot drive the BER below the floor. All is not lost, however. If the "BER floor" is low enough (as it is in this case), then a good error correcting code (such as the LDPC code defined in the iNET standard) can remove the errors to produce a close-to-error-free link. 

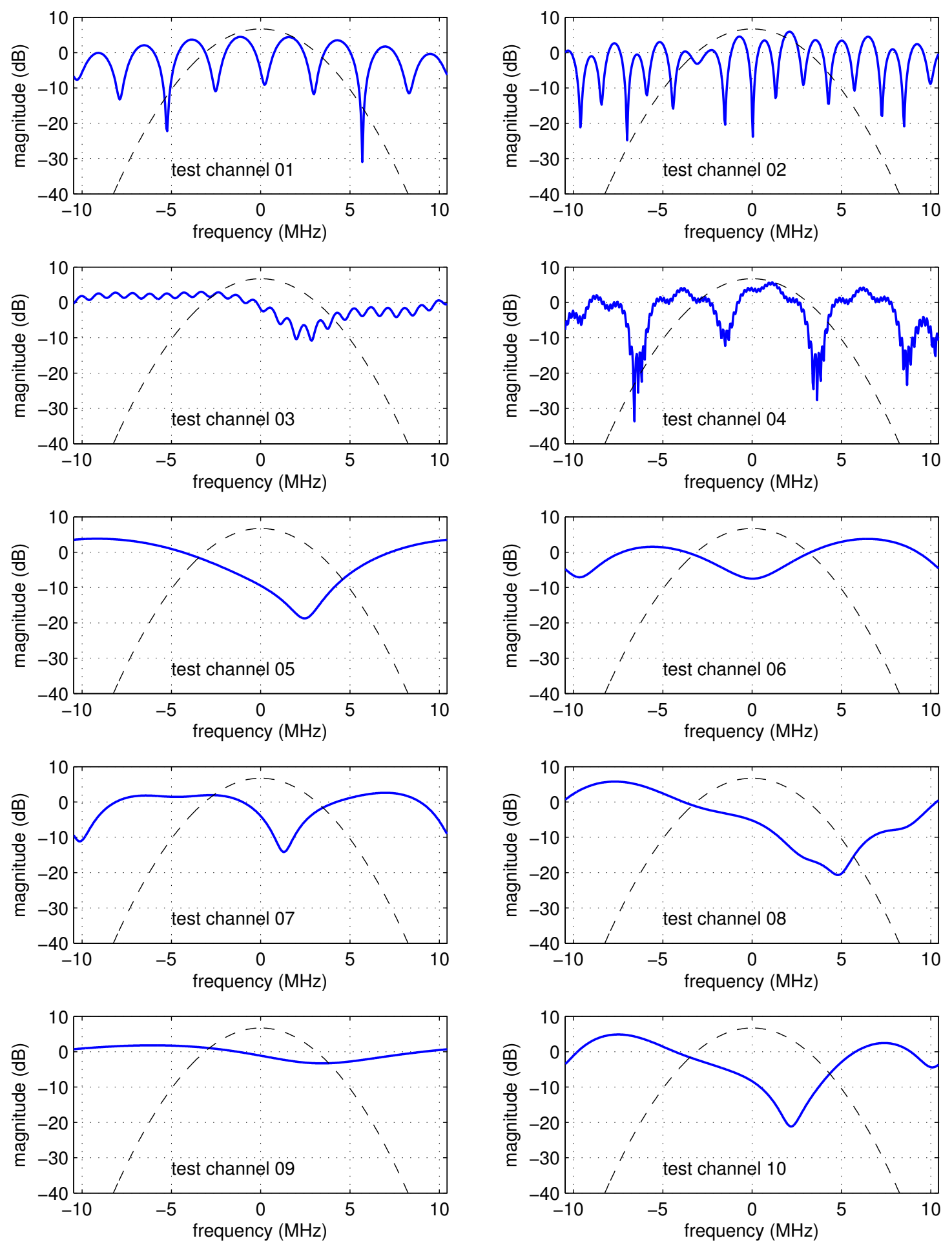

Figure 5: Frequency-domain plots of the example channels from channel sounding experiments at Edwards AFB. In each plot, the thick line is the channel frequency response and the thin line is the power spectral density of SOQPSK-TG operating at $10.3125 \mathrm{Mbits} / \mathrm{s}$. 
Table 1: Description of the ten test channels used in the simulations.

\begin{tabular}{crrrl}
\hline channel & $N_{1}$ & $N_{2}$ & length & environment \\
\hline 01 & 1 & 7 & 9 & Taxiway E \\
02 & 2 & 17 & 20 & Taxiway E \\
03 & 1 & 22 & 24 & Taxiway E \\
04 & 5 & 13 & 19 & Takeoff on 22L \\
05 & 1 & 1 & 3 & Cords Road \\
06 & 1 & 2 & 4 & Cords Road \\
07 & 0 & 4 & 5 & Cords Road \\
08 & 2 & 3 & 6 & Black Mountain \\
09 & 1 & 1 & 3 & Black Mountain \\
10 & 2 & 3 & 6 & Land on 22L \\
\hline
\end{tabular}
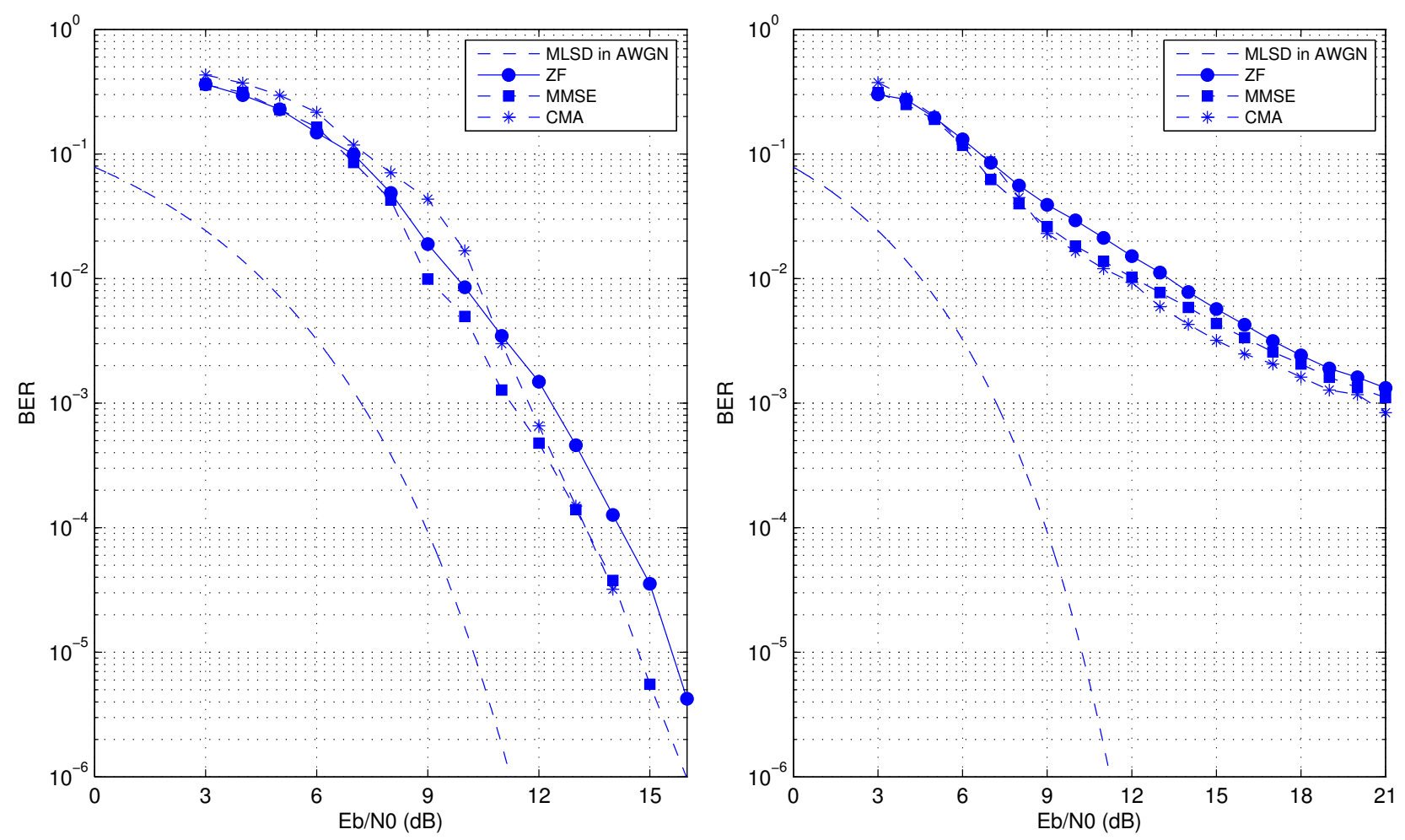

Figure 6: Simulation results for test channel 1 (left) and test channel 2 (right). 

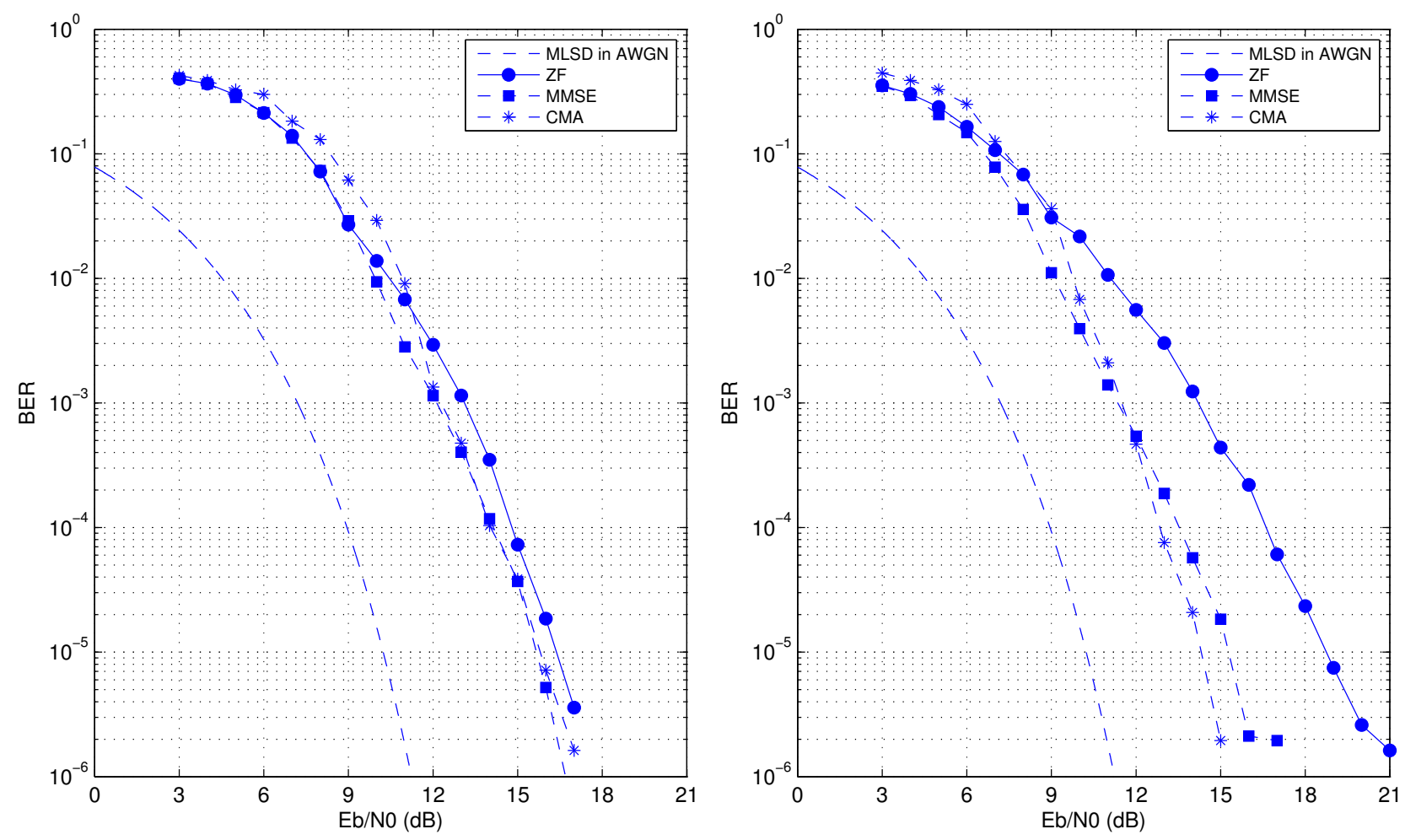

Figure 7: Simulation results for test channel 3 (left) and test channel 4 (right).
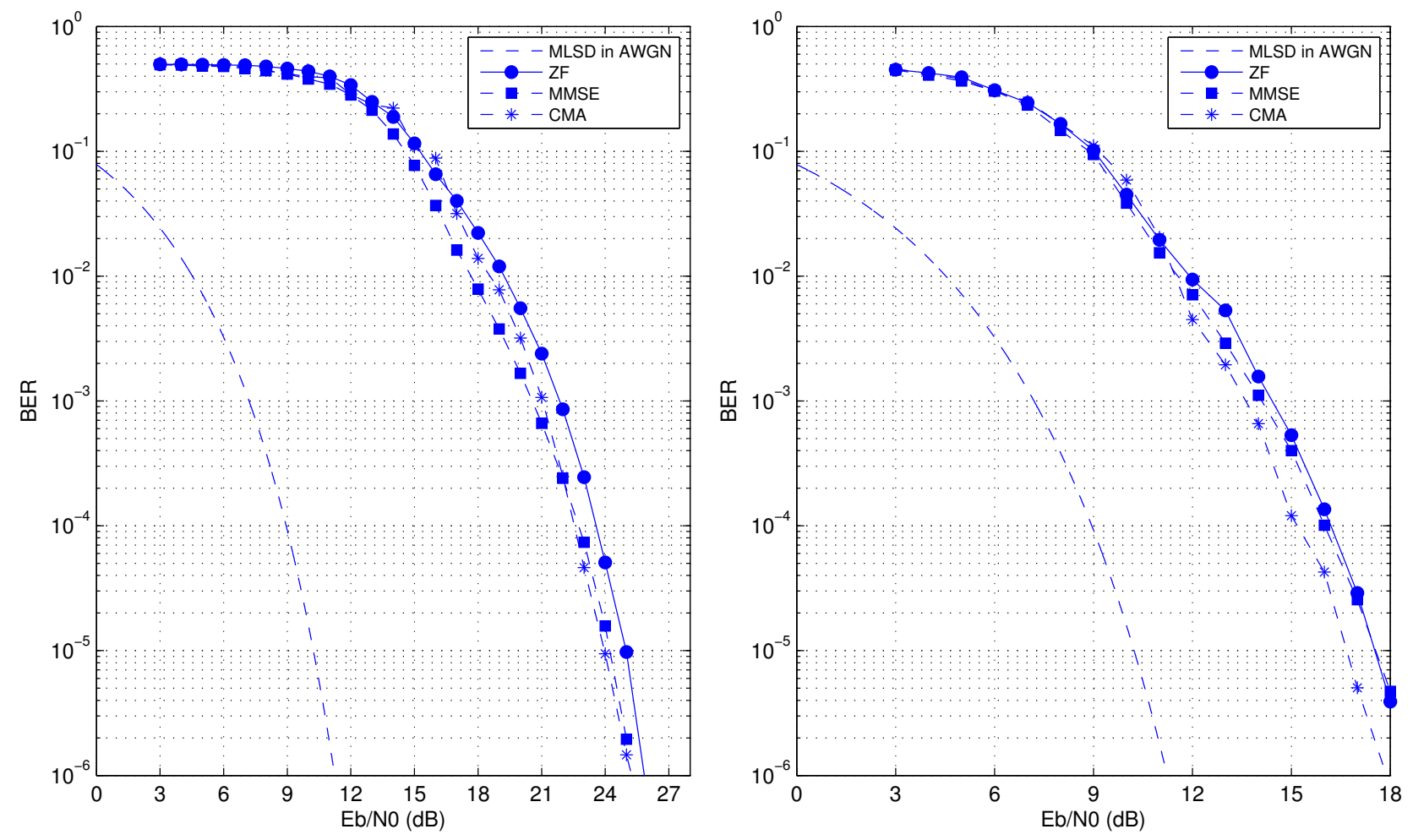

Figure 8: Simulation results for test channel 5 (left) and test channel 6 (right). 

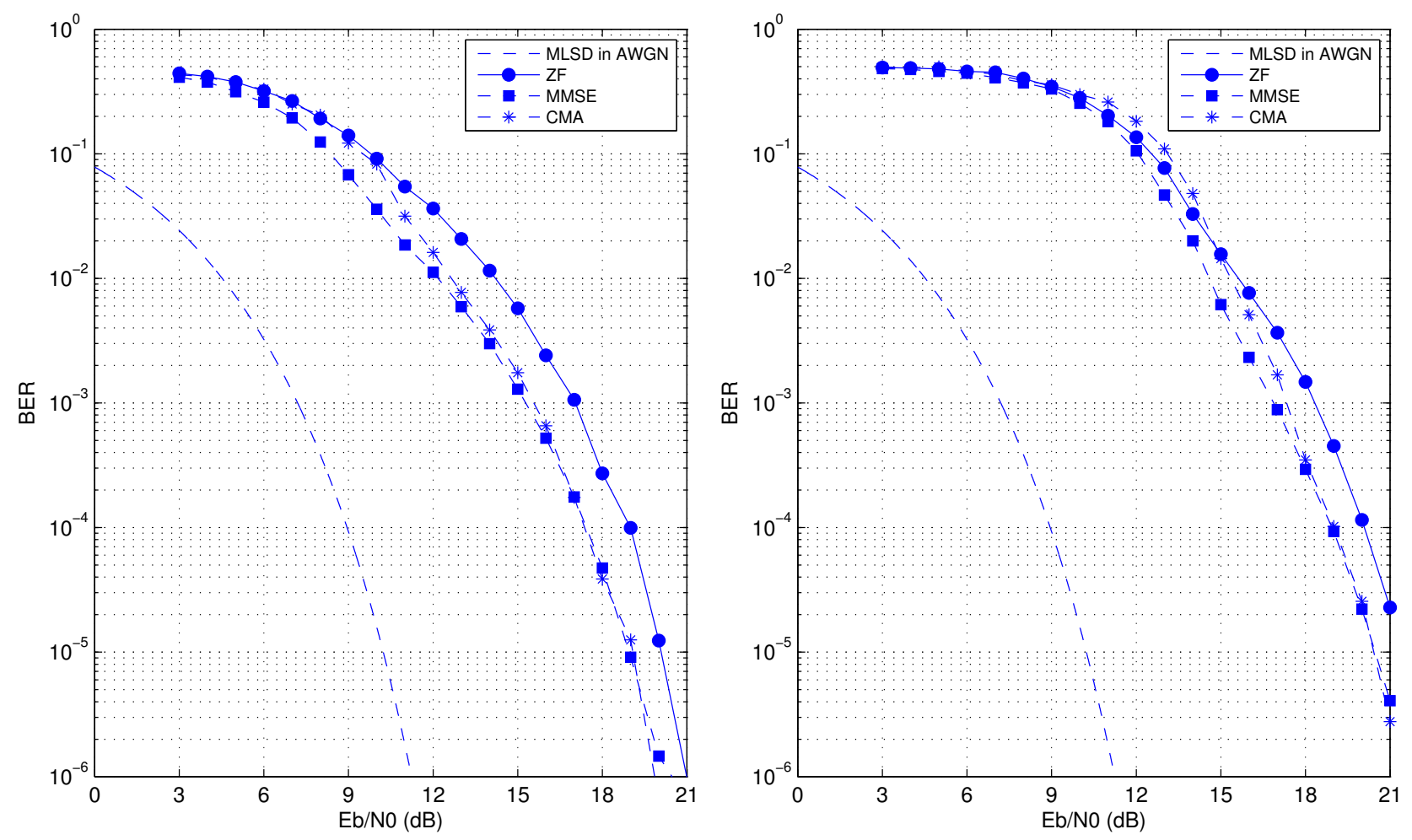

Figure 9: Simulation results for test channel 7 (left) and test channel 8 (right).
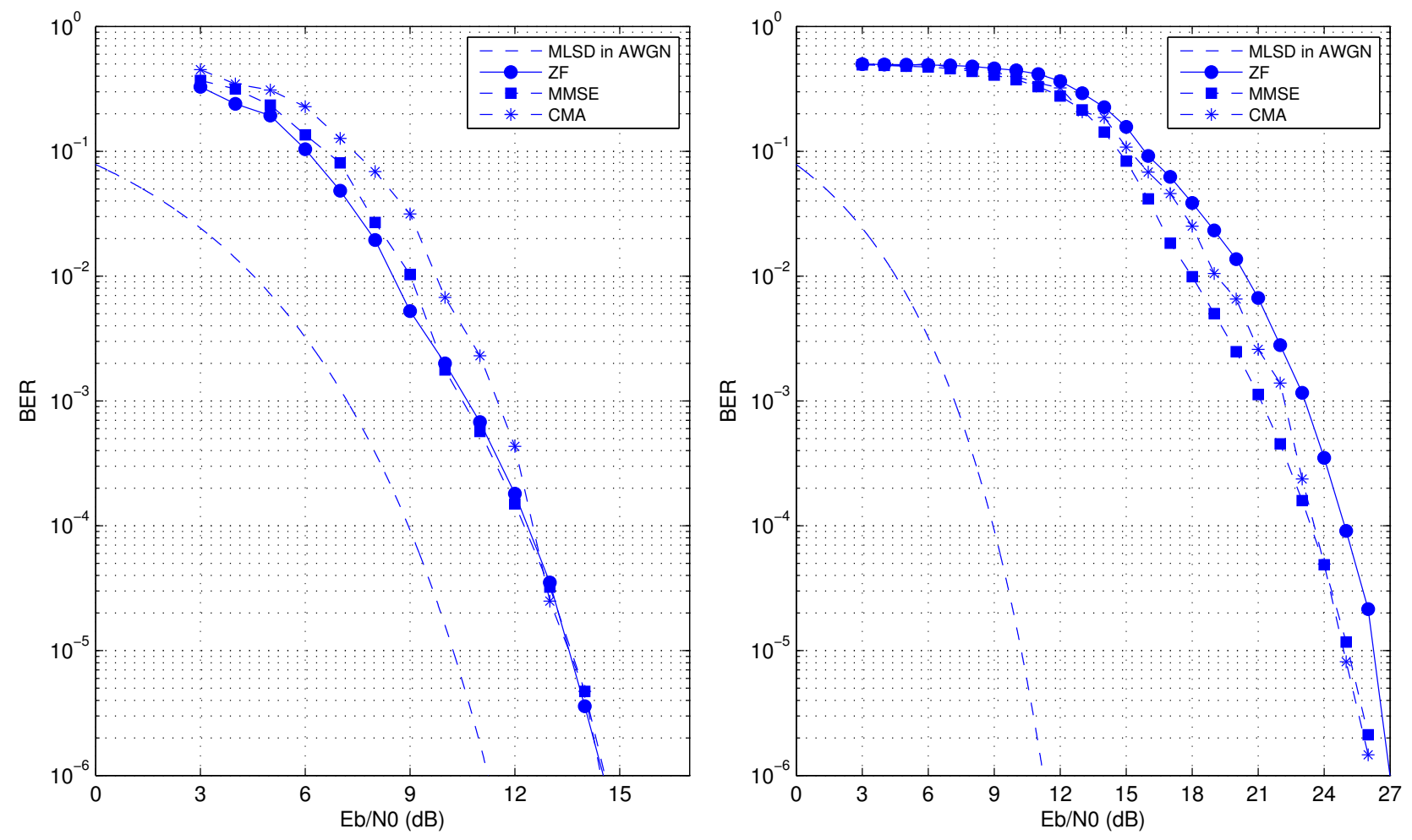

Figure 10: Simulation results for test channel 9 (left) and test channel 10 (right). 


\section{CONCLUSIONS}

This paper has demonstrated the effectiveness of the ZF, MMSE, and CMA equalizers with iNETformatted SOQPSK-TG. The equalization algorithms leverage the existence of known bit sequences in the preamble and ASM fields of the iNET packet to realize data-aided equalizers. The effectiveness of these equalization techniques over ten test channels, derived from channel sounding experiments at Edwards AFB, was evaluated. The BER curves for test channels 1, 3-10 display the desirable "waterfall" shape, whereas the BER curve for test channel 2 displays a "BER floor." Fortunately, the "BER floor" is low enough to allow error correcting codes, such as the iNET LDPC code to correct the errors and provide virtually error-free performance.

\section{ACKNOWLEDGEMENTS}

This work was funded by the Test Resource Management Center (TRMC) Test and Evaluation Science and Technology (T\&E/S\&T) Program through the U.S. Army Program Executive Office for Simulation, Training and Instrumentation (PEO STRI) under contract W900KK-13-C-0026 (PAQ).

\section{REFERENCES}

[1] Z. Ye, E. Satorius, and T. Jedrey, "Enhancement of advanced range telemetry (ARTM) channels via blind equalization," in Proceedings of the International Telemetering Conference, Las Vegas, NV, October 2001.

[2] T. Hill and M. Geoghegan, "A comparison of adaptively equalized PCM/FM, SOQPSK, and multi-h CPM in a multipath channel," in Proceedings of the International Telemetering Conference, San Diego, CA, October 2002.

[3] M. Geoghegan, "Experimental results for PCM/FM, Tier I SOQPSK, and Tier II Multi-h CPM with CMA equalization," in Proceedings of the International Telemetering Conference, Las Vegas, NV, October 2003.

[4] E. Law, "How well does a blind adaptive CMA equalizer work in a simulated telemetry multipath environment?" in Proceedings of the International Telemetering Conference, San Diego, CA, October 2004.

[5] M. Rice, M. Saquib, and E. Perrins, "Estimators for iNET-formatted SOQPSK-TG," in Proceedings of the the International Telemetering Conference, San Diego, CA, October 2014.

[6] integrated Network Enhanced Telemetry (iNET) Radio Access Network Standards Working Group, "Radio access network (RAN) standard, version 0.7.9," Tech. Rep., available at https://www.tena-sda.org/display/INET/iNET+Platform+Interface+Standards.

[7] J. Proakis and M. Salehi, Digital Communications, 5th ed. New York: McGraw-Hill, 2008. 
[8] A. McMurdie, M. Rice, and E. Perrins, "Preamble detection for iNET-formatted SOQPSKTG," in Proceedings of the the International Telemetering Conference, San Diego, CA, October 2014.

[9] T. Nelson, E. Perrins, and M. Rice, "Near optimal common detection techniques for shaped offset QPSK and Feher's QPSK,' IEEE Transactions on Communications, vol. 56, no. 5, pp. 724-735, May 2008.

[10] E. Perrins, "FEC systems for aeronautical telemetry," IEEE Transactions on Aerospace and Electronic Systems, vol. 49, no. 4, pp. 2340-2352, October 2013.

[11] M. Hayes, Statistical Digital Signal Processing and Modeling. New York: John Wiley \& Sons, 1996.

[12] M. Rice, M. S. Afran, and M. Saquib, "Equalization in aeronautical telemetry using multiple antennas," submitted to IEEE Transactions on Aerospace \& Electronic Systems, 2014.

[13] M. Rice and M. Jensen, "A comparison of L-band and C-band multipath propagation at Edwards AFB," in Proceedings of the International Telemetering Conference, Las Vegas, NV, October 2011. 